\title{
Use of Facial Recognition System in Modern World for Human Welfare
}

\author{
Ravindra Kumar
}

\begin{abstract}
The increasing interconnection in the world now presents the customers with customization on delivery of a product, service, and experience. The increasing interconnection is recording a very high rise and there is a challenge on ensuring that the service and the product delivery is stable. However, artificial intelligence has availed a solution to the stabilization and has been a solution to the modern world problems. Artificial intelligence has achieved the development of facial recognition technology without messing up with citizen's rights and firms.
\end{abstract}

Keywords: The increasing interconnection is recording a very high rise and there is a challenge on ensuring that the service and the product delivery is stable.

\section{INTRODUCTION}

A fter much hard work on ant attention, a google famous laboratory was able to announce their breakthrough by creating facial recognition, artificial intelligence model. The technology uses image recognition to do digital imaging on the physical appearance and creates a private database to store the profile. The system is dormant until future inputs match the stored images, which triggers pre-determined responses (Facial Recognition Technology Explained, n.d.). The artificial intelligence model can identify human faces in both their existence and the specific individual to which the face belongs.

\section{FACIAL RECOGNITION SOFTWARE}

$\mathrm{F}_{\text {acial recognition software is historically working like }}$ other forms of biometric identification such as voice recognition, iris recognition, and fingerprint identification. Facial recognition works such that it can analyze particular photographs or any other biometric data and identify the specific marks within the mark (Soltanpour, 2017). The comparison aspects in the face are similar to the comparison of lines in the fingerprint identification but facial identification is a little complicated than fingerprint identification. If the model identifies a critical threshold that is similar between the samples and the example patterns, a declaration of a match is done. The facial recognition, however, denies the match if the sample and the existing

Manuscript received on July 29, 2021.

Revised Manuscript received on September 21, 2021.

Manuscript published on September 30, 2021

* Correspondence Author

Ravindra Kumar*, Technical Account Manager, Navvis Healthcare, St. Louis MO, 63021. Email: rakumar25@gmail.com

(c) The Authors. Published by Blue Eyes Intelligence Engineering and Sciences Publication (BEIESP). This is an open access article under the CC BY-NC-ND license (http://creativecommons.org/licenses/by-nc-nd/4.0/) patterns differ from each other, the match is not declared in this process.

\section{FACIAL RECOGNITION SOFTWARE AND ALGORITHMS}

There is an emergence in a variety of image identification methods which has made it a lot easier. Gradients This involves the process of replacement of the image to be identified with a version that is made up of the most relevant features that match the facial identification. Gradients replace each pixel with a representative of how the brightness of the pixel compares to the pixels around it. The measure of pixel brightness eases the recognition of the face across a variety of lighting situations. This will help identify how the face looks in different brightness samples and the match will be declared if it matches. The relativity of lighting attribute holds in between the shots, objective lighting tends to be more variable than relative lighting.

\section{PROJECTION}

The other process includes the wrapping of the face around the third dimension using a projection of $2 \mathrm{D}$ and $3 \mathrm{D}$ photos which makes the identification in flat and static images difficult in 2D and 3D such as when wrapped on a cylinder (Soltanpour, 2017). On completion of this preparation, the system does face encoding or collapsing such that the distinguishing characteristics and patterns are made smaller. The simplification of the file exists only to conduct cross-checking on the current and other encoded faces. Hence, when shown a photo of Leonardo DiCaprio, the system first wraps and analyzes the photo, generates an encoded version of the photo, and conducts a comparison against other encoded faces with the current encoded face The basis to compare the facial matches are the stored faces. The stored information is always linked to the data of the matched face, the data includes; names and addresses, the stored faces are pre-associated with the data.

\section{USE CASES FOR FACIAL RECOGNITION SYSTEMS}

\section{A. Human Safety and general Security}

A facial recognition system is applied in the modern world as an identity model to; identity, conduct an interdiction, and the capturing of criminal suspects. The facial identification model allows the users to scan and recognize certain faces, the targeted face can be applied and the system scans every face until the desired output is attained. An example of the case is when the New York police, in 2019, used the facial recognition mechanism to track a rapist down and arrested him.

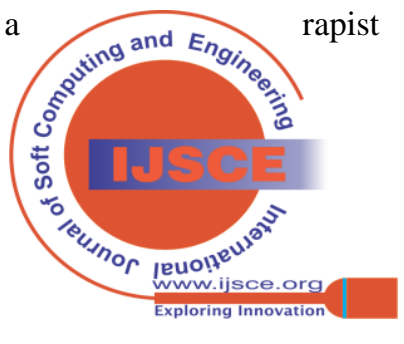




\section{Use of Facial Recognition System in Modern World for Human Welfare}

The facial recognition identified the alleged's face in a certain place and he was tracked down by the police. However, facial recognition is best applied in national border checks, the facial recognition mechanism can identify missing children which will help arrest human traffickers and find the victims. Swift lane is among the fastest-growing facial recognition door access model (Woodward Jr, 2003).

\section{B. Convenience}

When the facial recognition mechanism is applied in various access control systems, facial recognition will provide a passive, and a doubtless solution. The keypad combination won't be the major stress among users. The system will use facial detection, this process just needs that the user to spend some time looking at a designated facial recognition camera. Facial recognition does not need the insertion of a finger or anything else and thus easing the requirements to access the system. The system is rendered as a no-touch system and the door is configured to open immediately the face matches the one that is in the system (Nguyen, 2017).

\section{Customer Service}

Facial recognition mechanism in businesses and trading activities. Producers, distributors, consumers, and other trading platforms that use the facial recognition mechanism to tailor the client's experience and cater to VIPs, include the provision of concierge service. An example of a store that uses the service is Amazon GO stores, the store uses facial recognition to create a simpler check-out process in the client system.

\section{Healthcare Advances}

Specialists at the National Human Genome Research Institute have utilized facial acknowledgment programming to make an exact determination of DiGeorge Syndrome, a chromosomal issue that causes numerous wellbeing challenges. Analysts thought about 156 Latin Americans, Africans, Caucasians, and Asians with DiGeorge Syndrome against others without the infirmity. Facial acknowledgment yielded a practically $97 \%$ pace of right analysis. Other medical services applications incorporate an application that ensures patients accept their prescription as recommended (Soltanpour, 2017). Furthermore, associations are utilizing it in the fight against Coronavirus. For instance, CLEAR, the organization that assists explorers through security in U.S. air terminals, is carrying out an instrument that utilizes facial acknowledgment to decide if a worker has Covid and should remain at home.

\section{CONCLUSION}

Facial recognition mechanism is the current trend in terms of security and other identification solution in the modern world. The mechanism is widely spread across the world. The increasing interconnection is recording a very high rise and there is a challenge on ensuring that the service and the product delivery is stable. However, artificial intelligence has availed a solution to the stabilization and has been a solution to the modern world problems. Facial recognition software is historically working like other forms of biometric identification such as voice recognition, iris recognition, and

fingerprint identification. Facial recognition works such that it can analyze particular photographs or any other biometric data and identify the specific marks within the mark. The facial recognition mechanism is growing and the future of facial recognition shows that there shall be no decrease in its usage.

\section{REFERENCES}

1. Facial Recognition Technology Explained. (n.d.). Retrieved from Summa Linguae Technologies: https://summalinguae.com/language-technology/facial-recognition-tech nology-explained/

2. Nguyen, D. T. (2017). Person recognition system based on a combination of body images from visible light and thermal cameras. Sensors, 17(3), 605.

3. Soltanpour, S. B. (2017). A survey of local feature methods for 3D face recognition. Pattern Recognition, 72, 391-406.

4. Woodward Jr, J. D. (2003). Biometrics: A look at facial recognition. RAND CORP SANTA MONICA CA

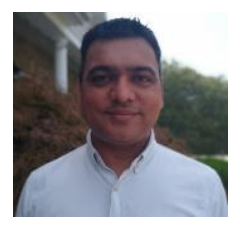

\section{AUTHOR'S PROFILE-}

Ravindra Kumar, is a technologist in the healthcare IT, population health management, clinical software applications product management, and Implementation space. Ravindra Kumar is interested in social use of Artificial Intelligence and spending his time in researching how to find ways using AI to save human life and make human life better and easy. He has over fifteen years of proven experience in leading and making globa teams spanning various functions like Technical, Solutions Architecture, Technical Alliances, Development, and Quality. He is passionate about value-based care and is actively involved in designing and implementing new approaches for data-driven initiatives for population health management for Medicare Advantage and ACO markets. In Past, Ravindra was involved in end to end implementation of Medicare Advantage plans, and Population health IT systems. He has good knowledge of care management, Utilization Management and other applications for value based care. He has a great interest in healthcare data analytics and is pretty comfortable in using Tableau, SQL, PowerBI for analysis of various healthcare data (Claims, eligibility, provider data, etc), including EMRs, HL7, ADT and FHIR records. He also loves to train people in quality practices, including RPA(Robotic Process Automation), Test Automation, and Agile engineering.

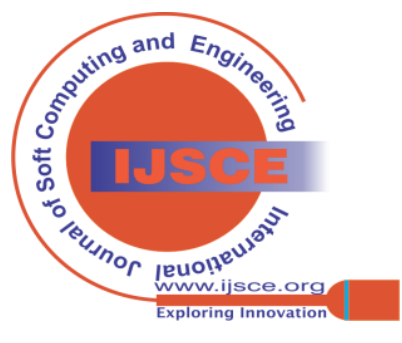

